\title{
Gênero Pfaffia: aspectos químicos, farmacológicos e implicações para o seu emprego terapêutico
}

\author{
Rates, S.M.K.*; Gosmann, G. \\ Departamento de Produção de Matéria-Prima, Faculdade de Farmácia, \\ Universidade Federal do Rio Grande do Sul, Porto Alegre, RS
}

Resumo: Os relatos encontrados na literatura científica sobre a composição química e atividades farmacológicas do gênero Pfaffia spp. são apresentados. Os estudos sobre esse gênero são ainda bastante escassos, sendo assim, o uso racional de espécies de Pfaffia, com finalidade terapêutica ainda depende de conhecimento aprofundado de suas propriedades farmacológicas e do desenvolvimento de tecnologias de produção e controle de qualidade.

Unitermos: Pfaffia; Panax ginseng; ginseng-brasileiro; fáfia; composição química; atividades farmacológicas.

ABSTRACT: Pfaffia: available chemical and pharmacological data and their implications for its therapeutical use. This review summarizes available chemical and pharmacological data about Pfaffia genre. Scientific studies about these species are still lacking, and their therapeutical rational use depends on the availability of more informations about their pharmacological properties and the development of production and control quality technologies.

Key words: Pfaffia spp.; Panax ginseng; ginseng-brasileiro; fáfia; chemical data; pharmacological data.

No Brasil, várias espécies do gênero Pfaffia, família Amaranthaceae, são utilizadas em substituição ao ginseng, sendo conhecidas como "ginseng-brasileiro" e fáfia. Entre essas espécies são citadas: Pfaffia glomerata (Spreng.) Pedersen, Pfaffia iresinoides Sprengel e Pfaffia paniculata (Mart.) Kuntze (SHIOBARA et al., 1992). P. paniculata está entre os maiores volumes de venda da indústria nacional de fitoterápicos (COMISSÃO, 1995). A exemplo do ginseng, o uso de Pfaffia tornou-se uma panacéia, sendo popularmente denominada "paratudo", "suma" ou "corango" (OLIVEIRA, 1986). Os produtos são, geralmente, rotulados como $P$. paniculata ou ginseng-brasileiro e as indicações fornecidas pelos fabricantes são: "revigorante, regenerador celular, indicado para o esgotamento físico e mental e falta de memória" ou "indicado como auxiliar no tratamento de irregularidades circulatórias, estresse, anemia, diabetes..." ou "indicado nos casos de fadiga física, intelectual e de indisposição em geral; contribui no tratamento da fraqueza sexual."

O termo ginseng pode se referir a muitas plantas diferentes. O ginseng da Coréia, Panax ginseng C.A. Meyer, é considerada a espécie típica; no entanto, outras espécies são utilizadas, como: Panax japonicus C.A. Meyer, Panax quinquefolium L., Panax trifolium L., Panax zingiberensis 
C.Y. Wu e Feng e Eleutherococcus senticosus Maxim. Todas são espécies muito semelhantes, pertencentes a família Araliaceae, cujos rizomas e raízes são utilizados em preparações classificadas como agentes adaptógenos, isto é, contêm substâncias com ação não específica, normalizadora das funções do organismo (HOSTTETMANN e MARSTON, 1995). Essas preparações têm origem na medicina chinesa, mas são comercializadas nos Estados Unidos, e também no Brasil, como suplemento alimentar.

No entanto, enquanto a espécie Panax ginseng é provavelmente a mais popular e bem conhecida planta medicinal documentada até hoje, os estudos sobre o gênero Pfaffia são, ainda, bastante escassos, se considerarmos a extensão de seu uso.

Assim, monografias de ginseng constam na Martindale $32^{\text {nd }}$ (PARFITT, 1999), na lista da Comissão E e da OMS (BLUMENTHAL, 1998), com as indicações principais de tônico e revigorante, em casos de fadiga, debilidade e convalescença. Critérios de qualidade para as raízes são definidos pela Farmacopéia Alemã (DEUTSCHES, 1998), Farmacopéia Britânica (BRITISH, 1996ab), Farmacopéia Francesa (PHARMACOPÉE, 1982) e Farmacopéia Japonesa (PHARMACOPOEIA, 1982). Os efeitos farmacológicos mais bem documentados são antiestresse, imunomodulatório e inibitório da agregação plaquetária. O ginseng parece apresentar uma baixa incidência de efeitos adversos, porém estes não podem ser ignorados, sendo considerados como principais a hipertensão e o potencial efeito estrogênico. Em usuários crônicos, ou que consomem altas doses, pode ocorrer o desenvolvimento da "Síndrome de Abuso do Ginseng", com hipertensão, nervosismo, insônia, erupções cutâneas e diarréia matinal. A dose recomendada não é superior a dois gramas do pó da raiz (padronizado em 1,5\% de ginsenosídeos expressos como ginsenosídeo $\mathrm{Rg} 1$ ), ou o equivalente a isto, diariamente, por um período consecutivo máximo de três meses. $O$ ginseng é contra-indicado para pessoas saudáveis abaixo de 40 anos de idade; pessoas com distúrbios de pressão arterial, especialmente hipertensão; mulheres com irregularidade menstrual; pessoas com distúrbios da coagulação sangüínea e no período agudo de trombose coronária; diabéticos; asmáticos; pacientes com desordens psiquiátricas, assim como, durante a gravidez e amamentação. Entre as interações medicamentosas mais importantes estão aqueles com a fenelzina, estimulantes em geral, corticosteróides, hormônios esteróides, hipoglicemiantes, anticoagulantes, glicosídeos cardiotônicos, fármacos que alteram a pressão arterial e sedativos. Deve ser evitado o uso concomitante de ginseng com bebidas à base de cafeína e álcool etílico (BALDWIN et al., 1986; WONG et al., 1998, e literatura citada).

Muitos compostos foram isolados das partes subterrâneas do ginseng, principalmente saponinas, conhecidas como ginsenosídeos, cujo teor nas raízes pode variar de 0,5 a 3\%. Quanto à composição química existem dois grupos de Panax: aquelas espécies que apresentam principalmente saponinas do tipo damarano- $P$. ginseng, $P$. quinquefolium e $P$. trifolium, e um segundo grupo que inclui as espécies $P$. japonicus e $P$. zingiberensis, possuindo majoritariamente saponinas derivadas do ácido oleanólico (HOSTTETMANN e MARSTON, 1995; SCHENKEL et al., 2002) (Figura 1).

As saponinas são apontadas como os constituintes ativos do ginseng e há um crescente interesse em sua caracterização e na sua investigação farmacológica. Contudo, o mecanismo de ação do ginseng não está completamente esclarecido. Provavelmente, seus efeitos são mediados pelo eixo hipotálamo-hipófise-adrenal, através do aumento dos níveis séricos de corticosterona (WONG et al., 1998). Além disso, muitos dos resultados até agora relatados mostram-se inconsistentes ou contraditórios. Algumas de suas frações químicas ou componentes apresentam ações opostas. Por exemplo, para o ginsenosídeo $\mathrm{Rb}_{1}$ são relatadas atividades hipotensora, analgésica, antipirética, sedativa do SNC, tranqüilizante, anticonvulsivante e antipsicótica, enquanto que o ginsenosídeo $\mathrm{Rg}_{1}$ mostra atividade hipertensora, antifadiga e estimulante do SNC, em baixas doses. Estes resultados conflitantes podem ser devido ao tipo de preparação empregada, 
via de administração, dose, presença ou ausência de compostos biologicamente ativos na amostra avaliada, entre outros fatores (FOSTER, 1996).

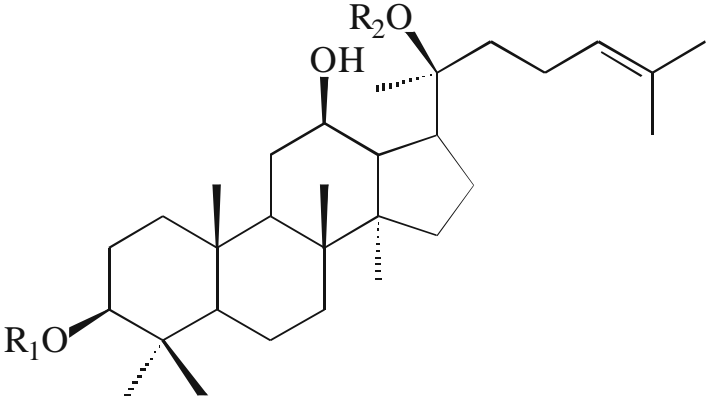

A - ginsenosídeo $\mathrm{Rb}_{1}$

(saponina com esqueleto do tipo damarano)

$\mathrm{R}_{1}=-\beta$-D-glicose- $(2-1)-\beta-\mathrm{D}-\mathrm{glicose}$

$R_{1}=-\beta-D-g l i c o s e-(6-1)-\beta-D-g l i c o s e$

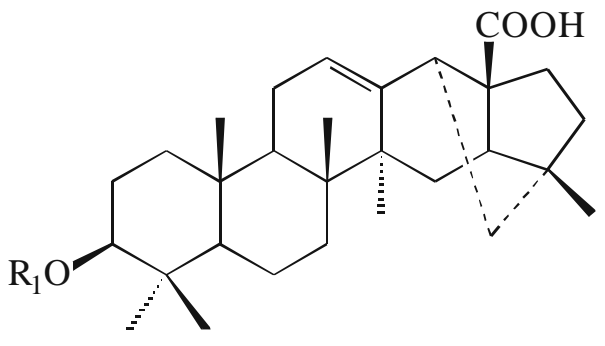

$C$ - ácido fáfico $\mathrm{R}_{1}=\mathrm{H}$

fafosídeo A R1 = - $\beta$-D-ácido glicurônico-(2-1)- $\beta$-D-xilose (saponina com esqueleto do tipo nortriterpênico)

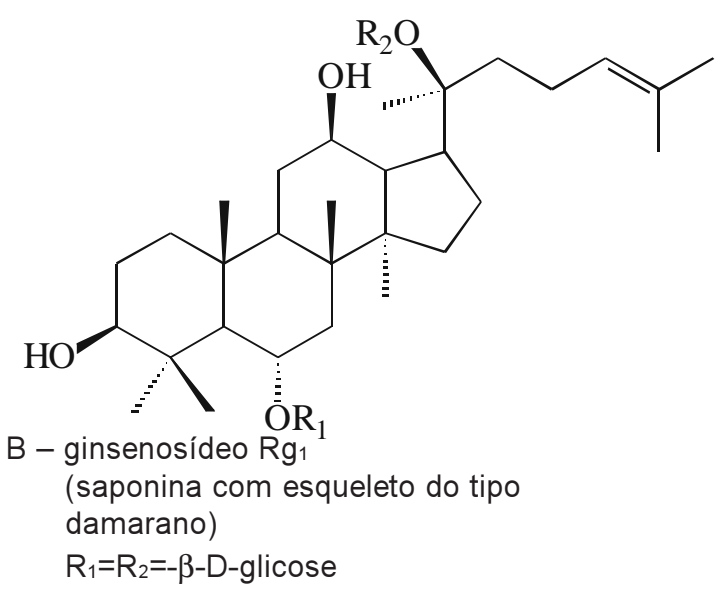<smiles>CC(C)(O)CCC(O)[C@H](O)C1CCC2(O)C3=CC(=O)[C@H]4CC(O)C(O)C[C@]4(C)C3CC[C@]12C</smiles>

D - ecdisterona

Figura 1. Principais constituintes químicos das espécies de Panax, Araliaceae $(A, B)$ e de Pfaffia, Amaranthaceae $(C, D)$.

O gênero Pfaffia possui 27 espécies distribuídas no Brasil (TANIGUCHI et al., 1997), sendo que a diferenciação das espécies não é simples e, muitas vezes, são encontradas no comércio substituições entre as espécies. A espécie $P$. paniculata, referida como substituta do ginseng, também no Rio Grande do Sul, é um vegetal de rara ocorrência nesse Estado, sendo nativas as espécies $P$. glomerata e $P$. tuberosa (VASCONCELLOS, 1982). De fato, em avaliações anteriores, foi possível detectar que algumas amostras comercializadas como $P$. paniculata em Porto Alegre (RS), possivelmente, eram P. glomerata (DE PARIS et al., 1998). As principais características morfológicas e micrográficas das raízes de $P$. glomerata e $P$. paniculata foram descritas e estão em vias de publicação, permitindo a identificação segura das mesmas (GOSMANN et al., 2002). Do mesmo modo, são encontradas algumas diferenças inter-específicas na composição química que podem definir perfis farmacológicos e toxicológicos distintos, além de auxiliar, também, na 
diferenciação das espécies (Tabela 1) (TAKEMOTO et al., 1983; NAKAl et al., 1984; NISHIMOTO et al., 1984, 1987; 1988; SHIOBARA et al., 1992, 1993a, b).

Como apresentado na Tabela 1, os fafosídeos, saponinas nortriterpênicas derivadas do ácido fáfico, e os ecdisteróides, em especial a ecdisterona, são encontrados em espécies de Pfaffia (Figura 1). As saponinas do gênero Pfaffia não estão ainda caracterizadas do ponto de vista da atividade biológica. Os fafosídeos A, C, D, E e F possuem potencial atividade antitumoral, e foram isolados apenas de P. paniculata (NAKAl et al., 1984; NISHIMOTO et al., 1984).

Tabela 1: Compostos isolados de espécies de Pfaffia

\begin{tabular}{|c|c|}
\hline ESPÉCIE & CONSTITUINTES QUÍMICOS \\
\hline P. glomerata & $\begin{array}{l}\text { ácido famérico, ácido glomérico, ácido oleanólico, ecdisterona, } \\
\text { rubrosterona, ?-D-glicopiranosil-oleanolato (SHIOBARA et al., } \\
\text { 1993a) }\end{array}$ \\
\hline P. iresinoides & $\begin{array}{l}\text { chikusetsusaponina IVa, ecdisterona, 25-O-?-D-glicopiranosil- } \\
\text { ecdisterona, iresinosídeo, 25-O-?-D-glicopiranosil-podecdisona } \\
\text { B, polipodina B, pterosterona, 24-O-? -D-glicopiranosil- } \\
\text { pterosterona (NISHIMOTO et al., 1987, 1988) }\end{array}$ \\
\hline P. paniculata & $\begin{array}{l}\text { ácido fáfico, alantoína, sitosterol, estigmasterol, fafosídeos A, B, } \\
\text { C, D, E, F, (TAKEMOTO et al., 1983; NAKAI et al., 1984; } \\
\text { NISHIMOTO et al., 1984) }\end{array}$ \\
\hline P. pulveru & $\begin{array}{l}\text { ácido fáfico, ácido pulvérico, ecdisterona, fafosídeo G, } \\
\text { pulverulactona, rubrosterona (SHIOBARA et al., 1992, 1993b) }\end{array}$ \\
\hline
\end{tabular}

Os relatos encontrados na literatura científica sobre as atividades farmacológicas deste gênero versam basicamente sobre a planta na forma de pó, como extrato bruto ou semi-purificado (MEDLINE/IPA, 1970-1999; ISI, 1945-2002/02) e estão apresentados resumidamente a seguir:

- P. paniculata: potencial atividade antitumoral do ácido fáfico e de fafosídeos (NAKAI et al., 1984; NISHIMOTO et al., 1984); reação alérgica (asma ocupacional) em trabalhadores envolvidos com o processamento da raiz (SUBIZA et al., 1991); extrato hidroalcoólico apresentou efeito analgésico do tipo antiinflamatório e toxicidade aguda baixa, em camundongos, via i.p. (MAZZANTI e BRAGHIROLI, 1994); efeito mutagênico do extrato aquoso detectado pelo teste de Ames em Salmonella typhimurium (RIVERA et al., 1994); efeito estimulante de extrato fluido, v.o., no desempenho sexual de ratos machos (ARLETTI et al., 1999); melhoria do estado de hidratação e propriedades reológicas de hemáceas falciformes (BALLAS, 2000); efeito inibitório de leucemia espontânea, em camundongos, do pó da planta v.o. (WATANABE et al., 2000); ausência de efeito mutagênico utilizando como sistemateste células de medula óssea de ratos, após tratamento agudo com pó da planta, via gavagem (SOUZA e VICENTINI, 2001). 
- P. iresinoides: efeito mutagênico do extrato aquoso detectado pelo teste de Ames em Salmonella typhimurium (RIVERA et al., 1994); o extrato aquoso e uma fração de saponinas, v.o., apresentaram efeito antiinflamatório em modelos de inflamação em ratos (TANIGUCHI et al., 1997).

- P. glomerata: nosso grupo de pesquisa vem trabalhando há alguns anos com $P$. glomerata, investigando a qualidade das preparações farmacêuticas disponíveis no mercado, estudando parâmetros para o controle da qualidade destas preparações e a farmacologia pré-clínica direcionada, principalmente, às ações sobre o sistema nervoso central. Os resultados obtidos sugerem que o extrato hidroalcoólico das partes subterrâneas de $P$. glomerata (500 mg/kg i.p.) apresenta uma atividade depressora do sistema nervoso central e efeito amnésico, contrariando a utilização popular desta espécie como estimulante (DE PARIS et al., 1998, 2000). Na continuação, o fracionamento bioguiado deste extrato foi iniciado, e os resultados indicam que a fração orgânica mais apolar é a fração ativa, apresentando atividade depressora em ambas as vias de administração testadas, v.o. e i.p. (FENNER et al., 2001, 2002). Estudos in vitro também demonstraram que extratos hidroalcoólicos das raízes e rizomas de $P$. glomerata não apresentaram efeito citotóxico sobre linhagens de células tumorais, ação antiviral sobre vírus do tipo Herpes humano, efeito antifúngico, nem ação inibidora sobre monoaminoxidases cerebrais (GOSMANN et al., 2002). MARQUES (1998) apresentou dados sobre a ação benéfica na aprendizagem e memória de ratos idosos em modelos crônicos, além de uma baixa toxicidade em roedores. Em humanos, o mesmo autor demonstrou que esse extrato melhorou o desempenho de voluntários idosos normais em testes psicométricos, particularmente, na memória de curto prazo e memória declarativa, mas causou prejuízo na praxia e orientação geral desses voluntários.

Uma avaliação cuidadosa dos dados apresentados acima indica que, embora existam estudos pré-clínicos para $P$. paniculata e mesmo estudos clínicos para P. glomerata, a eficácia e a segurança de seu uso ainda não estão plenamente definidas. Não há critérios publicados para a padronização e controle de qualidade dos produtos comerciais, como por exemplo, a definição da substância ativa ou compostos marcadores. Também, a substituição do ginseng por Pfaffia spp. não parece ser adequada, visto que a sua constituição química é diferente (Tabela 1) e que as propriedades biológicas das espécies de Pfaffia precisam ser melhor avaliadas.

Desta forma, o uso racional de Pfaffia com finalidade terapêutica ainda depende do conhecimento aprofundado de suas propriedades farmacológicas e do desenvolvimento de tecnologias de produção e controle de qualidade. Considerando a legislação de fitoterápicos vigente no País, a comercialização destes produtos, neste momento, só se justifica se for possível considerá-lo como produto fitoterápico tradicional, segundo a RDC ANVISA 17/2000 e Portaria SVS/MS 116/1996 (BRASIL, 1996, 2000).

\section{AGRADECIMENTOS}

As autoras agradecem às acadêmicas da Faculdade de Farmácia da UFRGS: Ana Paula Heckler, Elyara F. Pacheco, Gilda A. Neves e Raquel Fenner pela excelente colaboração técnica e à PROPESQ-UFRGS e ao CNPq pela concessão de bolsas de iniciação científica e financiamento para o estudo de espécies de Pfaffia da Região Sul do Brasil. 


\section{REFERÊNCIAS BIBLIOGRÁFICAS}

ARLETTI, R.; BENELLI, A.; CAVAZZUTI, E.; SCARPETTA, G.; BERTOLINI, A. Stimulating property of Turnera diffusa and Pfaffia paniculata extracts on the sexual behavior of male rats. Psychopharmacology, v.143, p.15-19, 1999.

BALDWIN, C.A.; ANDERSON, L.A.; PHILLIPSON, J.D. What pharmacists should know about ginseng? Pharmaceutical Journal, v. 237, p.583-586, 1986.

BALLAS, S.K. Hydration of sickle erythrocytes using a herbal extract (Pfaffia paniculata) in vitro. British Journal of Haematology, v.111, p.359-362, 2000.

BLUMENTHAL, M. (Ed.) The Complet German Comission E monographs: Therapeutic Guide to Herbal Medicines. Austin: American Botanical Council, 1998.

BRASIL. Ministério da Saúde. Secretaria de Vigilância Sanitária. Portaria n.116 de 08.08.1996. Diário Oficial da União, 12.08.1996.

BRASIL. Ministério da Saúde. Agência Nacional de Vigilância Sanitária. Resolução de Diretoria Colegiada (RDC) n.17 de 24.2.2000. Diário Oficial da União, 24.04.2000.

BRITISH Herbal Pharmacopoeia. Dorset, 1996a.

BRITISH Herbal Compendium. v.1. Dorset, 1996b.

COMISSÃO DE HOMEOPATIA E FITOTERAPIA DO SINDUSFARM-SP. Lista de Plantas SINDUSFARM. São Paulo: SINDUSFARM, 1995. p.9-13.

DE PARIS, F.; SALGUEIRO, J.; MOREIRA ROSA, R.; GOSMANN, G. IZQUIERDO, I. RATES, S.M.K. Análise cromatográfica e atividade depressora central de produtos vegetais comercializados como Pfaffia paniculatta Mart. Kuntze (Amaranthaceae) no RS (Brasil). In: VI Jornada de Jóvenes Investigadores de la AUGM. Santa Fé, Argentina, 1998. Resumenes, p.119.

DE PARIS, F.; SALGUEIRO, J.B.; QUEVEDO, J.; IZQUIERDO, I.; RATES, S.M.K. Psychopharmacological screening of Pfaffia glomerata Spreng (Amaranthaceae) in rodents. Journal of Ethnopharmacology, v.73, p.261-269, 2000.

DEUTSCHES Arzneibuch. Stuttgart: Deutscher Apotheker, 1998.

FENNER, R., HECKLER, A., PRIETSCH, F. L., NEVES, G., PACHECO, E. F., GOSMANN, G., RATES, S. M. K. Efeito depressor do Sistema Nervoso Central (SNC) do extrato orgânico de Pfaffia glomerata Spreng (Amaranthaceae). In: IX Jornada de Jóvenes Investigadores de la AUGM. Rosario, Argentina, 2001. Anales, p.234.

FENNER, R.; GATTUSO, M.; GATTTUSO, S.J.; HECKLER, A.P.M.; NEVES, G.; PACHECO, E.F.; GOSMANN, G.; RATES, S M.K. Análise Micrográfica e Avaliação Farmacológica de espécies de Pfaffia (Amaranthaceae). In: X Jornada de Jovens Pesquisadores da AUGM. Florianópolis, Brasil, 2002. Resumos, p.764.

FOSTER, S. Asian Ginseng, Panax ginseng. Austin: American Botanical Council, n.303, 1996.

GOSMANN; G.; GATTUSO; S.; GATTUSO; M.; FENNER, R.; PACHECO; E.F.; FERRAZ, A.; SAVI, L.A.; BARARDI, C.R.M.; SIMÕES; C.M.O.; SORTINO, M.; ZACCHINO; S.; GNERRE; C.; TESTA, B.; RATES, S.M.K. Botanical (morphological, micrographic), chemical and pharmacological characteristics of Pfaffia species (Amaranthaceae) native to South Brazil. Brazilian Journal of Pharmaceutical Sciences, São Paulo, submetido para publicação, 2002.

HOSTETTMANN, K.; MARSTON, A. Saponins- chemistry and pharmacology of natural products. Cambridge: University, 1995.

MARQUES, L.C. Avaliação da ação adaptógena das raízes de Pfaffia glomerata (Sprengel) PedersenAmaranthaceae. São Paulo, 145p. Tese (Doutorado). Universidade Federal de São Paulo. Psicobiologia. 1998. 
MAZZANTI, G.; BRAGHIROLLI, L. Analgesic and antiinflammatory action of Pfaffia paniculata (Martius) Kuntze. Phytotherapy Research, v.8, p.413-416, 1994.

NAKAI, S.; TAKAGI, N.; MIICHI, H.; HAYASHI, S.; NISHIMOTO, N.; TAKEMOTO, T.; KIZU, H. Pfaffosides, nortriterpenoid saponins, from Pfaffia paniculata. Phytochemistry, v.23, p.1703-1705, 1984.

NISHIMOTO, N., NAKAI, S., TAKAGI, N., HAYASHI, S., TAKEMOTO, T., ODASHIMA, S., KIZU, H., WADA, Y. Pfaffosides and Nortriterpenoid Saponins from Pfaffia paniculata. Phytochemistry, v.23, p.139-142, 1984.

NISHIMOTO, N.; SHIOBARA, Y.; FUJINO, M.; INOUE, S-S.; TAKEMOTO, T.; OLIVEIRA, F. de; AKISUE, G.; AKISUE, M. K.; HASHIMOTO, G.; TANAKA, O.; KASAI, R.; MATSUURA, H. Ecdysteroids from Pfaffia iresinoides and reassignment of some ${ }^{13} \mathrm{C}$ NMR chemical shifts. Phytochemistry, v.26, p.2505-2507, 1987.

NISHIMOTO, N.; SHIOBARA, Y.; INOUE, S-S.; FUJINO, M.; TAKEMOTO, T.; YEOH, C.L.; OLIVEIRA, F. de; AKISUE, G.; AKISUE, M. K.; HASHIMOTO, G. Three ecdysteroid glycosides from Pfaffia iresinoides. Phytochemistry, v.27, p.1665-1668, 1988.

OLIVEIRA, F. de. Pfaffia paniculata (Martius) Kuntze - o ginseng-brasileiro. Revista Brasileira de Farmacognosia, v.1, p.86-92, 1986.

PARFITT, K. (Ed.) Martindale- The Complete Drug Reference. 32 ${ }^{\text {nd }}$ ed.London: Pharmaceutical Press, 1999. p.1584.

PHARMACOPÉE Française. 10 $10^{\text {ème }}$ ed. Paris: Adrapharm, 1982.

PHARMACOPOEIA of Japan. $10^{\text {th }}$ ed. Tokyo: Society of Japanese Pharmacopoeia, 1982. Versão em inglês.

RIVERA, I.G.; MARTINS, M.T.; SANCHES, P.S.; SATO, M.I.Z.; COELHO, M.C.L.; AKISUE, M.; AKISUE, G. Genotoxicity assessment through the Ames test of medicinal plants commonly used in Brazil. Environmental Toxicology and Water Quality, v.9, p.87-93, 1994.

SCHENKEL, E.P.; GOSMANN, G.; ATHAYDE, M.L. Saponinas. In: SIMÕES, C.M.O.; SCHENKEL, E.P.; GOSMANN, G.; MELLO, J.C.P. de; MENTZ, L.A.; PETROVICK, P.R. Farmacognosia. Da planta ao medicamento. 4. ed. Porto Alegre: Editora Universidade; Florianópolis: Ed. da UFSC, p.301-330, 2002.

SHIOBARA, Y.; INOUE, S-S.; NISHIGUCHI, Y.; KATO, K.; TAKEMOTO, T.; NISHIMOTO, N.; OLIVEIRA, F. de; AKISUE, G.; AKISUE, M. K.; HASHIMOTO, G. Pfaffane-type nortriterpenoids from Pfaffia pulverulenta. Phytochemistry, v.31, p.1737-1740, 1992.

SHIOBARA, Y.; INOUE, S-S.; KATO, K.; NISHIGUCHI, Y.; OISHI, Y.; NISHIMOTO, N.; OLIVEIRA, F. de; AKISUE, G.; AKISUE, M. K.; HASHIMOTO, G. A nortriterpenoid, triterpenoids and ecdysteroids from Pfaffia glomerata. Phytochemistry, v.32, p.1527-1530, 1993a.

SHIOBARA, Y.; INOUE, S-S.; KATO, K.; NISHIGUCHI, Y.; NISHIMOTO, N.; OLIVEIRA, F. de; AKISUE, G.; AKISUE, M. K.; HASHIMOTO, G. Pfaffane-type nortriterpenoids from Pfaffia pulverulenta. Phytochemistry, v.33, p.897-899, 1993b.

SOUZA, V.H.; VICENTINI, V.E.P. Análise da ação mutagênica do ginseng brasileiro (Pfaffia paniculata) em ratos tratados via gavagem. In: $V$ Congresso da Sociedade Brasileira de Mutagênese, Carcinogênese e Teratogênese Ambiental. Gramado, RS, setembro, 2001. Livro de Resumos, p.76.

SUBIZA, J.; SUBIZA, J.L.; ESCRIBANO, P. M. HINOJOSA, M.; GARCIA, R. JEREZ, M.; SUBIZA, E. Ocupational asthma caused by Brazil ginseng dust. Journal of Allergy and Clinical Immunology, v.88, p.731736, 1991.

TANIGUCHI, S.F.; BERSANI-AMADO, C.A.; SUDO, L.S.; ASSEF, S.M.C.; OGA, S. Effect of Pfaffia iresinoides on the experimental inflammatory process in rats. Phytotherapy Research, v.11, p.568-571, 1997.

TAKEMOTO, T.; NISHIMOTO, N.; NAKAI, S.; TAKAGI, N.; HAYASHI, S.; ODASHIMA, S.; WADA, Y. Pfaffic acid, a novel nortriterpene from Pfaffia paniculata Kuntze. Tetrahedron Letters, v.24, p.1057-1060, 1983.

VASCONCELOS, J.M.O. Estudo taxonômico sobre Amaranthaceae no RS, Brasil. Porto Alegre. Dissertação de Mestrado. Curso de Pós-Graduação em Botânica, Universidade Federal do Rio Grande do Sul, 1982. 
WATANABE, T.; WATANABE, M.; WATANABE, Y.; HOTTA, C. Effects of oral adminstration of Pfaffia paniculata (Brazilian ginseng) on incidence of spontaneus leukemia in AKR/J mice. Cancer Detection and Prevention, v.24, p.173-178, 2000.

WONG, A.H.C.; SMITH, M.; BOON, H.S. Herbal remedies in psychiatric practice. Archives of General Psychiatry, v.55, p.1033-1044, 1998.

* Autor para Correspondência:

Profa. Dra. Stela M. K. Rates

Departamento de Produção de Matéria-Prima Faculdade de Farmácia

Universidade Federal do Rio Grande do Sul Av. Ipiranga, 2752

90.610-000 - Porto Alegre - RS

e-mail: ratessmk@farmacia.ufrgs.br 